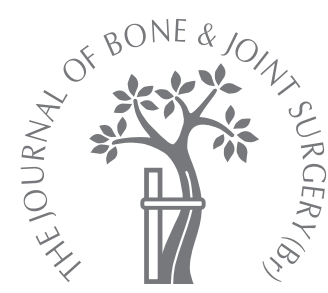

J. Victor, D. Van Doninck, L. Labey, F. Van Glabbeek, P. Parizel, J. Bellemans

From AZ St-Lucas, Bruges, Belgium

II. Victor, MD, Orthopaedic Surgeon

AZ St-Lucas, St-Lucaslaan 29 8310 Bruges, Belgium.

- D. Van Doninck, BSc, Medical Student

Catholic University, Oude Markt 13, Bus 5005, 3000 Leuven, Belgium.

- L. Labey, PhD, Research Engineer

European Centre for Knee Research, Technologiclaan 11, bis, 3001 Leuven, Belgium.

- F. Van Glabbeek, MD, PhD, Orthopaedic Surgeon

P. Parizel, MD, PhD, Chairman of Radiology

Antwerp University Hospital, Wilrijkstraat 10, 2650 Edegem, Belgium.

I. J. Bellemans, $\mathrm{MD}, \mathrm{PhD}$, Chairman of Orthopaedics University Hospitals, Herestraat 49, 3000 Leuven, Belgium.

Correspondence should be sent to Dr J. Victor; e-mail: j.victor@skynet.be

(C)2009 British Editorial Society of Bone and Joint Surgery doi:10.1302/0301-620X.91B5. $21827 \$ 2.00$

$J$ Bone Joint Surg $[\mathrm{Br}]$ 2009;91-B:683-90. Received 25 September 2008; Accepted after revision 20 January 2009

\title{
A common reference frame for describing rotation of the distal femur
}

\author{
A CT-BASED KINEMATIC STUDY USING CADAVERS
}

The understanding of rotational alignment of the distal femur is essential in total knee replacement to ensure that there is correct placement of the femoral component. Many reference axes have been described, but there is still disagreement about their value and mutual angular relationship. Our aim was to validate a geometrically-defined reference axis against which the surface-derived axes could be compared in the axial plane. A total of 12 cadaver specimens underwent $\mathrm{CT}$ after rigid fixation of optical tracking devices to the femur and the tibia. Three-dimensional reconstructions were made to determine the anatomical surface points and geometrical references. The spatial relationships between the femur and tibia in full extension and in $90^{\circ}$ of flexion were examined by an optical infrared tracking system.

After co-ordinate transformation of the described anatomical points and geometrical references, the projection of the relevant axes in the axial plane of the femur were mathematically achieved. Inter- and intra-observer variability in the three-dimensional CT reconstructions revealed angular errors ranging from $0.16^{\circ}$ to $1.15^{\circ}$ for all axes except for the trochlear axis which had an interobserver error of $2^{\circ}$. With the knees in full extension, the femoral transverse axis, connecting the centres of the best matching spheres of the femoral condyles, almost coincided with the tibial transverse axis (mean difference $-0.8^{\circ}$, SD 2.05). At $90^{\circ}$ of flexion, this femoral transverse axis was orthogonal to the tibial mechanical axis (mean difference $-0.77^{\circ}$, SD 4.08). Of all the surface-derived axes, the surgical transepicondylar axis had the closest relationship to the femoral transverse axis after projection on to the axial plane of the femur (mean difference $0.21^{\circ}$, SD 1.77). The posterior condylar line was the most consistent axis (range $-2.96^{\circ}$ to $-0.28^{\circ}, \mathrm{SD} 0.77$ ) and the trochlear anteroposterior axis the least consistent axis (range $-10.62^{\circ}$ to $+11.67^{\circ}$, SD 6.12). The orientation of both the posterior condylar line and the trochlear anteroposterior axis ( $p=0.001$ ) showed a trend towards internal rotation with valgus coronal alignment.

Poor outcomes and major complications after total knee replacement (TKR) have been linked directly to errors in rotational alignment of the components. ${ }^{1-5}$

Despite the clinical importance of correct rotational alignment the dilemma of choosing the correct surgical references remains unsolved. For the rotational alignment of the femoral component, two systems prevail. In the dependent resection, or tensioned-gaps technique, the surgeon performs ligamentous release to balance the knee in extension followed by resection of the posterior femoral surfaces parallel to the prepared cut tibial surface, after applying equal loads to the medial and lateral compartments. ${ }^{6,7}$ In the measured resection technique, a surface-derived reference axis of the femur is used as a guide to determine the position of the femoral component in the axial plane. Several different reference axes have been proposed including the posterior condylar line (PCL), 8,9 the surgical transepicondylar axis (surg TEA), ${ }^{10-12}$ the anatomical transepicondylar axis (anat TEA) ${ }^{13}$ and the trochlear anteroposterior axis (TRAx). ${ }^{14}$

There are four obstacles to surgical consistency as follows: semantic confusion over the definition of the 'correct axis', the natural variability of the reference axes, the inter- and intra-observer variability in the intra-operative determination of these references and the practical execution of the cuts.

There is a distinct difference between the 'desired' alignment in the axial plane of the femoral component and the 'natural' alignment of the distal femur. This difference can be explained by the fact that the normal natural tibial plateau has a varus configuration. ${ }^{15}$ The 
perpendicular coronal cut of the tibia will change this angle. Consequently, the femoral component will not be correctly aligned to provide an adequate flexion gap if it follows the natural surface anatomy. An external rotational compensation to the same degree as the correction of the tibial cut in the coronal plane will generally be advocated. Therefore since the literature refers to the 'correct' alignment of the axial plane of the femoral component, we always refer to the 'adapted' alignment of the femoral component in the axial plane, which differs from the natural situation.

The numerous studies which have compared axes, relevant for describing rotational alignment, have all used the PCL, surg TEA, anat TEA and TRAx in their examinations. ${ }^{9-14,16-19}$ These axes have been employed because the surface points describing them are palpable during surgery. From a methodological standpoint, however, the results are difficult to compare, given the absence of a fixed reference frame.

We wished to add more information to improve the understanding of the three-dimensional relationships of the distal femur by including geometrically defined reference axes, based on anatomical descriptions of the distal femur ${ }^{20}$ and the proximal tibia. ${ }^{21}$ In addition, we have used the dependent relation between the femur and tibia in extension and flexion to validate these geometrical axes, respectively called the femoral transverse axis (FTAx) and tibial transverse axis (TTAx).

Our aim was threefold:

1. To determine the natural angular variability of previously studied surface-derived axes (PCL, anat TEA, surg TEA, TRAx), compared with the FTAx, projected on the axial plane of the femur. The surface-derived axis with the smallest variability is theoretically the most useful axis for surgical reference.

2. To measure the angle between the FTAx and TTAx projected on the axial plane of the femur with the knee extended. Since there is no rotational freedom between the tibia and femur in full extension, this is a reliable position to measure the rotational relation between the two bones. If they are parallel in the axial plane, these axes can be validated as reliable rotational landmarks.

3 . To determine the relationship between the aforementioned femoral axes and the tibial mechanical axis at $90^{\circ}$ of flexion. It is hypothesised that the FTAx will be perpendicular to this tibial mechanical axis, indicating neutral varus/ valgus alignment at $90^{\circ}$ of flexion.

\section{Materials and Methods}

With the approval of the ethical committee, 12 cadaver limbs showing no signs of prior trauma or surgery, ligament imbalance or arthritis were disarticulated at the level of the hip in ten subjects (one matched pair, ten non-matched). The specimens were deep frozen and optical stereotactic reference frames were rigidly attached. Volumetric CT scans on a 64-row multidetector CT scanner (General Electric Lightspeed VCT, Milwaukee, Wisconsin) were performed using the following settings: $120 \mathrm{kVp}, 450 \mathrm{~mA}$; rotation time 0.5 ; pitch $0.516 / 1$, speed $20.62 \mathrm{~mm}$ per rotation; helical thickness $1.25 \mathrm{~mm}$; interval $0.8 \mathrm{~mm}$. The CT scan data were fed into a three-dimensional software system (Mimics 11.02 and its MedCAD module; Materialise, Haasrode, Belgium) for further determination of the relevant axes and surface points. Important surface landmarks were identified, using the quantitative morphological description by LaPrade et al. ${ }^{22,23}$ Geometrically important points such as the centre of the hip or the centre of the femoral condyles were determined by fitting a sphere in the outer surface mask of the respective structures.

A repeatability analysis was done on the first six specimens to evaluate the reliability of localising the bony landmarks used to define the four major rotational axes and the FTAx. Three observers (DVD, LL and JV) used bonesurface reconstructions of the scanned joints to identify the landmarks three times at a minimum interval of one week (for intra-observer precision) and independently (for interobserver precision). Based on the recorded differences in position for each landmark between repeated measurements and between observers, the angular deviation for each axis with respect to its mean orientation was calculated. Methodological analysis was carried out as described by Bland and Altman. ${ }^{24}$

The Cartesian co-ordinate system of the CT data was transformed into a new co-ordinate system for the femur and the tibia. The axial plane of the femur (or horizontal plane in a standing subject) was defined as the plane perpendicular to the mechanical axis of the femur and comprising the centre of the knee. The latter was defined as the most posterior point of the trochlear groove (top of the femoral notch). The FTAx connects the centres of the two best matching spheres of the medial and lateral femoral condyle. The anat TEA connects the medial epicondyle with the lateral epicondyle. The surg TEA connects the medial sulcus with the lateral epicondyle. The TRAx, commonly referred to as Whiteside's line ${ }^{19}$ connects the cranial and caudal deepest point of the trochlear groove (Fig. 1). At the tibia, the centre of the medial and lateral condyles were computed according to the description by Cobb et al, ${ }^{21}$ fitting the best matching circle to the cortical outline of the proximal tibia, $20 \mathrm{~mm}$ below the tibial spines. The line connecting the medial and lateral condylar centres, defined as the 'anatomical tibial axis' by Cobb et al, ${ }^{21}$ was referred to as the TTAx to maintain consistent terminology with the femur (Fig. 2). The cadaver specimens were moved from full extension to $90^{\circ}$ of flexion, while the optical stereotactic reference frames were followed by five previously calibrated infrared cameras (Vicon Motion Systems, Los Angeles, California). Since the stereotactic optical frames were rigidly attached to the femur and the tibia, and their spatial relation to the important reference points and axes on the bones was documented by the CT scan, the position of all points and axes on the femur relative to the tibia could be computed. 


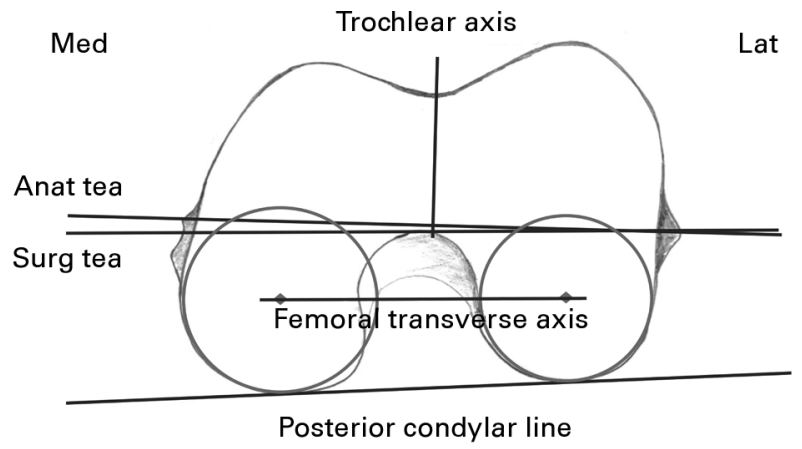

Fig. 1

Diagram showing the axes which have been studied in relation to the rotational alignment of the distal femur. The posterior condylar line $(P C L)$ is the tangent of the posterior part of the medial and lateral condyle. The anat TEA connects the medial epicondyle to the lateral epicondyle and the surg TEA the medial sulcus to the lateral epicondyle. The trochlear axis (Whiteside's line) connects the deepest point of the trochlear to the highest point in the notch, with the femur in caudal view.

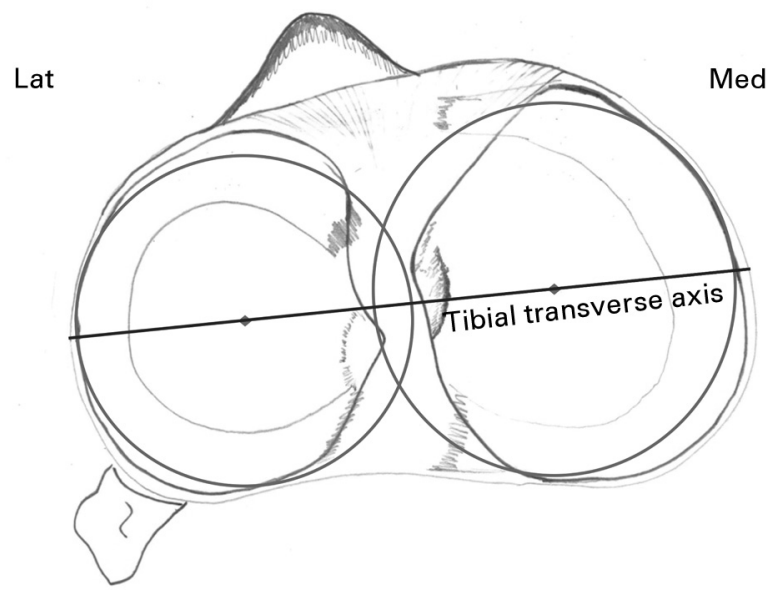

Fig. 2

Diagram showing the TTAx as defined by Cobb et al. ${ }^{21}$

The projection of the FTAx and TTAx on the axial plane was performed with the specimens in full extension (Fig. 3). The relation between the tibial mechanical axis and the various femoral axes was computed with the knees in $90^{\circ}$ of flexion (Fig. 4).

Statistical analysis. All datasets were checked for normal distribution. Pairwise comparisons between the different axes were performed with Statistica software, (Statsoft Inc., Tulsa, United States) using Student's $t$-test. Correlations were evaluated using Pearson's test. Statistical significance was set at a $\mathrm{p}$-value $\leq 0.05$.

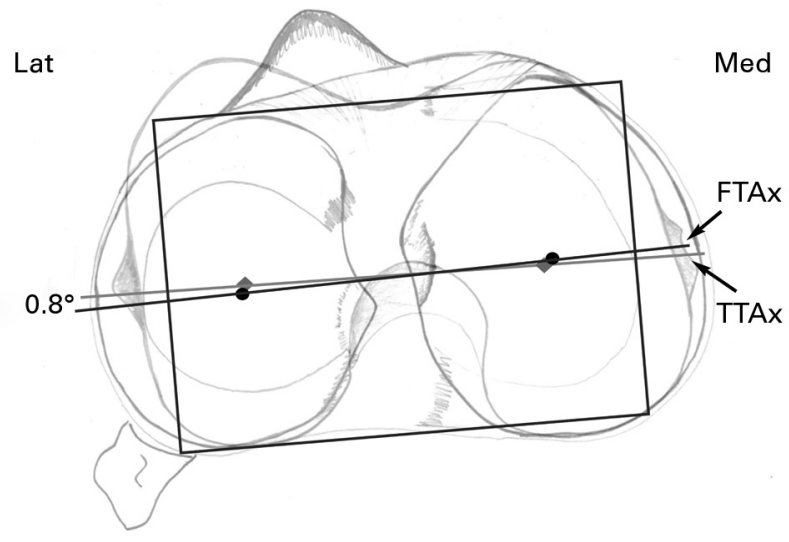

Fig. 3

Diagram showing the projection of the mean TTAx and the mean FTAx on the axial plane of the femur. The projection of both axes in the extended knee virtually coincide.

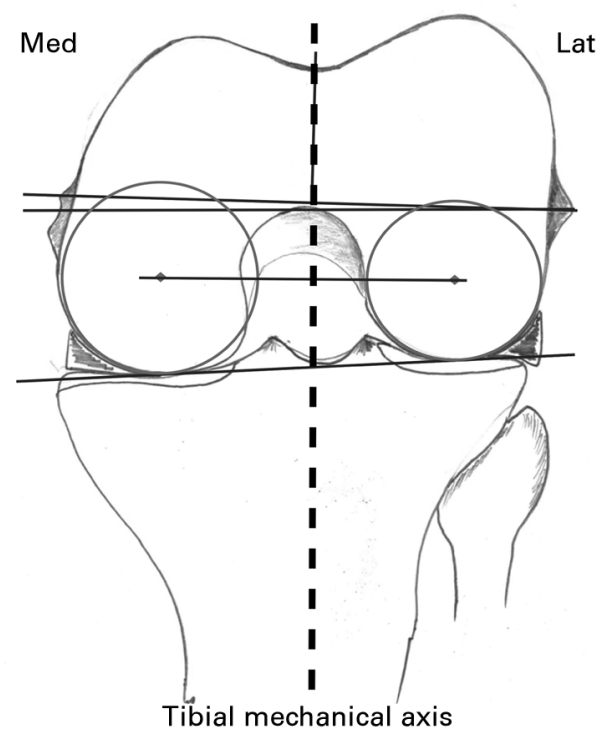

Fig. 4

Diagram showing the relation between the tibial mechanical axis and the distal femur at $90^{\circ}$ of flexion.

\section{Results}

Intra- and interobserver variability. We found a high level of reproducibility for both intra- and inter-observer error in our method. A repeatability analysis was done on the first six specimens to evaluate the precision of marking the four surface derived rotational axes and the femoral transverse axis. Three observers used bone surface reconstructions of 
Table I. Mean intra- and interobserver angular differences for the five femoral axes in degrees

\begin{tabular}{llllll}
\hline & FTAx & PCL & AnatTEA & SurgTEA & TRAx \\
\hline Intra & 1.15 & 0.16 & 0.52 & 0.57 & 1.24 \\
Inter & 1.15 & 0.57 & 1.15 & 1.15 & 2.07 \\
\hline
\end{tabular}

Table II. Angles in degrees, formed between the four major rotational axes of the distal femur and the FTAx. Negative values indicate a relative internal rotation of the variable in relation to the FTAx, positive values a relative external rotation of the variable to the FTAx

\begin{tabular}{lllll}
\hline \multicolumn{5}{c}{ Angle relative to the FTAx } \\
\cline { 2 - 5 } SpL & Anat TEA & Surg TEA & Perpendicular to TRAx \\
\hline 1 & -0.28 & 3.44 & 1.10 & -0.53 \\
2 & -1.23 & 4.44 & 1.52 & 1.23 \\
3 & -1.00 & 6.16 & 3.31 & -0.06 \\
4 & -1.02 & 2.22 & -1.73 & 4.69 \\
5 & -2.96 & 1.73 & 0.95 & -7.21 \\
6 & -1.18 & 5.23 & -0.76 & -0.71 \\
7 & -0.39 & 3.21 & 0.42 & 5.65 \\
8 & -2.01 & 0.35 & -2.91 & 0.03 \\
9 & -2.22 & 1.86 & -2.00 & -10.62 \\
10 & -1.44 & 3.26 & 0.81 & 6.08 \\
11 & -1.93 & 4.54 & 1.59 & 6.41 \\
12 & -1.22 & 4.34 & 0.24 & 11.67 \\
Mean & $-1.41^{*}$ & $3.40^{*}$ & $0.21^{*}$ & 1.39 \\
SD & 0.77 & 1.66 & 1.77 & 6.12 \\
\hline * p $<0.005$ & & & &
\end{tabular}

the scanned joints to identify the landmarks three times (for intra-observer precision) and independently (for interobserver precision). Based on the recorded differences in position for each landmark between repeated measurements and between observers, the mean angular difference for each axis can be calculated. ${ }^{24}$ The results are shown in Table I.

Variability and discrimination of the surface-derived axes. The projections on the axial plane of the resulting angles between the four major rotational axes and the FTAx per specimen are given in Table II. The surg TEA was almost parallel to the FTAx with a mean relative external rotation of $0.21^{\circ}$ (SD 1.77). The anat TEA had a mean external rotation relative to FTAx of $3.40^{\circ}$ (SD 1.66). The PCL had a mean relative internal rotation of $-1.41^{\circ}(\mathrm{SD}+0.77)$. The perpendicular to the trochlear axis had a mean external rotation of $1.39^{\circ}$ (SD 6.12).

The angle between the PCL and the FTAx had the least variability with an $\mathrm{SD}$ of $0.77^{\circ}\left(-2.96^{\circ}\right.$ to $\left.-0.28^{\circ}\right)$. The highest variability was found in the angle between the perpendicular to the TRAx and the FTAx with an SD of $6.12^{\circ}$ $\left(10.62^{\circ}\right.$ to $\left.+11.67^{\circ}\right)$ (Table II). Comparison of the mean values for the relative angles between the FTAx and the PCL, the surg TEA and the anat TEA showed clear distinctions with p-values of $<0.005$ for the PCL/anat TEA and anat TEA/surg TEA and $\mathrm{p}$-values $=0.008$ for the PCL/surg TEA ( $t$-tests). By contrast, comparison between the FTAx and the perpendicular to TRAx showed poor discrimination from the other axes because of the high SD (TRAx/PCL $\mathrm{p}=0.012 ;$ TRAx/anat TEA $\mathrm{p}=0.251 ;$ TRAx/surg TEA $\mathrm{p}=0.512$, all $t$-test) (Fig. 5). A strong correlation was found between the coronal alignment in full extension (defined as the angle between the femoral mechanical and tibial mechanical axis in the frontal plane) and the trochlear axis orientation $(r=0.81, p=0.001)$ indicating an increasing internal rotation of the TRAx orientation with increasing valgus. A weak correlation was found with the PCL orientation $(r=0.36, p=0.257)$ and no correlation with the $\operatorname{surg}(r=0.02, p=0.95)$ or anat TEA $(r=-0.01, p=0.97)$. Angle between the projected FTAx and TTAx at full extension. The projected centre of the medial condyle on the axial plane was found to be a mean of $1.6 \mathrm{~mm}(-2.6$ to -5.9$)$ anterior to the tibial transverse axis, compared with a mean of $2.83(-6.1$ to +0.66$)$ posterior to the projected centre of the lateral femoral condyle. This resulted in a mean angle between the FTAx and TTAx of $0.8^{\circ}\left(-1.8^{\circ}\right.$ to $\left.+3^{\circ}\right)$ (Fig. 3).

Relation between the femoral axes and the tibial mechanical axis at $90^{\circ}$ of flexion. For the analysis of the tibiofemoral alignment in relation to the distal femoral geometry, the angle between the tibial mechanical axis and the femoral rotation axes was computed. A graphical illustration of the statistics is shown in Figure 6 and the results are summarised in Table III.

The tibial mechanical axis was close to the perpendicular to the FTAx, surg TEA and parallel to the TRAx at $90^{\circ}$ of flexion. The perpendicular to the PCL of the femur was at a mean of $2.17^{\circ}$ of internal rotation relative to the TMAx. 


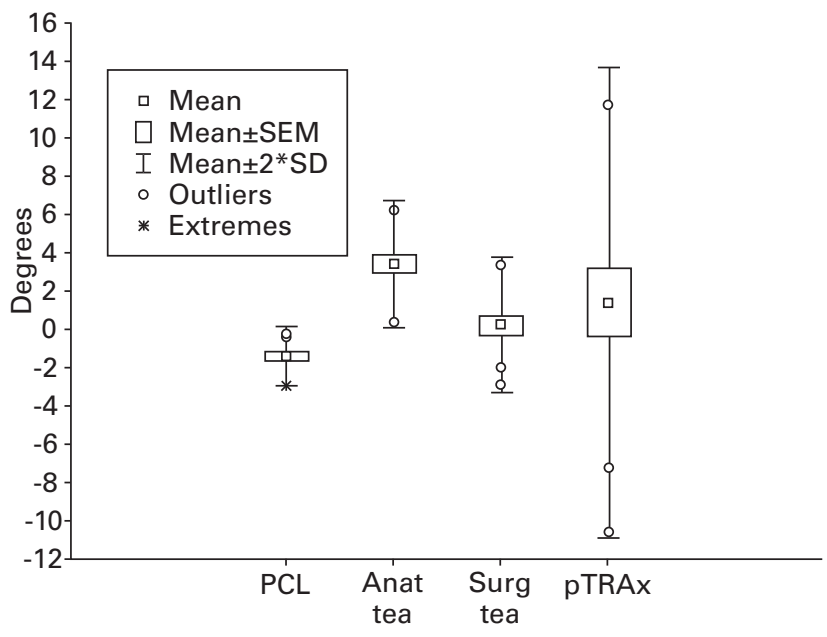

Fig. 5

Box-and-whisker plots of the four axes, relative to FTAX clearly showing the small spread of the posterior condylar line (PCL) and the large interindividual variability of the trochlear axis.

As expected, based upon the distal femoral geometrical values, the greatest variability was found in the angle between TMAx and TRAx (SD 5.96 $6^{\circ}$, range $-6.86^{\circ}$ to $\left.+12.7^{\circ}\right)$. There was a significant difference at $\mathrm{p}<0.005$ between the means for FTAx/PCL, FTAx/anat TEA, PCL/anat TEA and surg TEA/anat TEA ( $t$-test, all $\mathrm{p}<0.005)$. No significant difference was found between FTAx surg TEA $(p=0.93)$ and between perpendicular to TRAx/surg TEA $(\mathrm{p}=0.52)$.

\section{Discussion}

One of the most important errors leading to revision in TKR is malalignment. ${ }^{25}$ Small errors may be acceptable and may not interfere with the function of a TKR. ${ }^{4}$ In regard to rotational malalignment of the femoral component, excessive internal rotation has been related to pain, stiffness and patellar instability ${ }^{1,2,4,26-29}$ whereas excessive external rotation has been associated with flexion instability, ${ }^{30}$ increased shear forces on the patella ${ }^{5}$ and varus alignment in flexion. ${ }^{16}$

Unfortunately, the surgical process of positioning the implant is subject to errors. Failure to align a component properly with a desired axis can occur at three different levels. Occasionally, the desired reference axis such as the femoral mechanical axis, is not visible in vivo and a secondary axis, generally the centre of the intramedullary canal, is chosen to serve as a guide during surgery. Ideally, this secondary axis has a reliable angular relation, in statistical terms a small SD, to the desired axis. A first level of error is the individual variability in the angular relation between the desired axis and the surgical secondary axis. The second level of error is related to the intra-operative determination of the secondary surgical axis, in other words, the ability of the surgeon to locate accurately and reproducibly anatomical landmarks which lead to the secondary surgical axis.

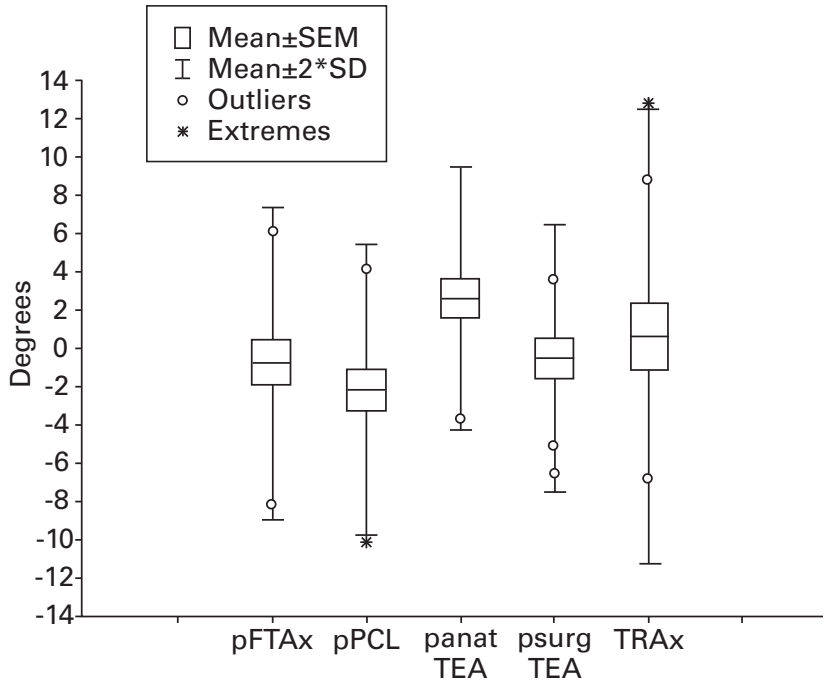

Fig. 6

Box-and-whisker plots of perpendiculars to the FTAx (pFTAx). The posterior condylar line (PCL), the anat TEA (point TEA, the surg TEA (psurg TEA) and the TRAx (pTRAx) relative to the tibial mechanical axis at $90^{\circ}$ of flexion.

Previous studies have emphasised the difficulties surgeons face in this area. ${ }^{31,32}$ The third level of error is related to the positioning and fixation of the cutting block and the execution of the cut with the saw. The subject of our study is at the first level, namely, the definition of the desired axis and its relation to the secondary surgical axis.

The main weakness of our study was the small sample size, caused by the extensive technical set-up and lengthy duration of the experiment. However, the distribution of the coronal alignment of the specimens fell within the range of that of previously published series, ${ }^{15}$ suggesting that it was a representative sample.

We decided to compare the position of the proposed desired axis of the femur (FTAx) to the tibia, since a description of the rotation of the distal femur is intrinsically related to the tibia. In the extended knee, we hypothesised parallelism in the axial plane between the projected FTAx and TTAx. In the flexed knee at $90^{\circ}$, we hypothesised that the tibial mechanical axis was perpendicular to the FTAx, resulting in neutral varus/valgus alignment. ${ }^{16}$

As shown in the results and illustrated in Figure 3, the FTAx and TTAx virtually coincide as they are projected on the axial plane of the femur with the knee in extension. This is a strong indication that the TTAx, the anatomical tibial axis of Cobb et al, ${ }^{21}$ is a reliable landmark for describing the rotation of the tibia and the FTAx for the femur. In addition, at $90^{\circ}$ of flexion, the mean value for the extension of the tibial mechanical axis crossing the FTAx was almost a right angle, indicating neutral alignment. This was a second argument for accepting the FTAx as a correct anatomical reference to describe the neutral rotation of the femur and to guide the surgeon in the placement of the femoral component during TKR. 
Table III. Angles in degrees, formed between TRAx and the perpendiculars to the FTAx, PCL, anatTEA and surgTEA and the tibial mechanical axis. Negative values indicate a relative internal rotation of the variable in relation to the TMAx, positive values a relative external rotation of the variable to the TMAx.

\begin{tabular}{|c|c|c|c|c|c|}
\hline \multirow[b]{2}{*}{ Specimen } & \multicolumn{5}{|c|}{ Angle relative to TMA in degrees at $90^{\circ}$ of flexion } \\
\hline & Perpendicular to FTAx & Perpendicular to $\mathrm{PCL}^{*}$ & Perpendicular to anat TEA & Perpendicular to TEA & TRAx \\
\hline 1 & -3.96 & -4.24 & -0.52 & -2.86 & -4.49 \\
\hline 2 & -4.48 & -5.71 & -0.04 & -2.96 & -3.25 \\
\hline 3 & -1.12 & -2.12 & 5.04 & 2.19 & -1.18 \\
\hline 4 & -3.37 & -4.39 & -1.15 & -5.10 & 1.32 \\
\hline 5 & 2.01 & -0.95 & 3.74 & 2.96 & -5.20 \\
\hline 6 & -0.55 & -1.73 & 4.68 & -1.31 & -1.26 \\
\hline 7 & -3.10 & -3.49 & 0.11 & -2.68 & 2.55 \\
\hline 8 & 6.11 & 4.10 & 6.46 & 3.20 & 6.14 \\
\hline 9 & 3.76 & 1.54 & 5.62 & 1.76 & -6.86 \\
\hline 10 & 2.72 & 1.28 & 5.98 & 3.53 & 8.80 \\
\hline 11 & -8.24 & -10.17 & -3.70 & -6.65 & -1.83 \\
\hline 12 & 1.03 & -0.19 & 5.37 & 1.27 & 12.70 \\
\hline Mean & 0.77 & -2.17 & 2.63 & -0.55 & 0.62 \\
\hline $\mathrm{SD}$ & 4.08 & 3.79 & 3.46 & 3.48 & 5.96 \\
\hline
\end{tabular}

With this FTAx as a reference, the surface derived 'secondary surgical' axes can be examined. The results presented in Table I and Figure 5 indicate that the PCL, the anat TEA and the surg TEA can be discriminated in the axial plane projection, based on their different orientation in individuals. The PCL displays the smallest interindividual variability with an SD of less than $1^{\circ}$. It is internally rotated relative to the anat and surg TEA. This is in line with previous published findings. ${ }^{9-14,17,19}$

Of the two transepicondylar axes, the axial plane projection, the surg TEA, is almost parallel to the FTAx. The anat TEA is consistently more externally rotated than the surg TEA, in our results relatively by a mean of $3.18^{\circ}$. This again confirms previously published work. ${ }^{11,12,19}$

Concerns can be raised about the trochlear axis (Whiteside's line). Despite the fact that the mean value of this axis shows an almost orthogonal orientation to the FTAx, the range $\left(-10.62^{\circ}\right.$ to $\left.+11.67^{\circ}\right)$ is considerable. If used as a single reference for the femoral component, it could lead to unacceptable outliers in rotational alignment. In addition, a significant correlation was found between the amount of 'internal' rotation of the trochlear axis and the valgus alignment in the coronal plane, which could limit the value of this reference in the valgus setting. This contrasts with the original description of this trochlear axis by Arima et al, ${ }^{14}$ in which they proposed this axis for the knee with valgus deformity. However, that study did not use valgus knees but 30 normal femora obtained from cadavers. No further information on coronal alignment was provided. They reported a range for the angle between the PCL and TRAx as $-1^{\circ}$ to $+10^{\circ}$. In our study we found a range between the PCL and TRAx of $-8.4^{\circ}$ to $12.9^{\circ}$. Three knees with valgus alignment form the lower end of this range (specimen 1, 5 and 9, Table II), indicating they had, relative to FTAx, more internal rotation of TRAx than of the PCL. Talbot and
Bartlett ${ }^{31}$ recently compared the orientation of the anterior surface of the femur, just cranial of the trochlea, with the TRAx in the axial plane and reported a reliable correlation between them but a significant variability with the PCL. They deduced that the TRAx was a reliable axis for rotational alignment. Without devaluing their contribution, their conclusion clearly illustrates how the finding of a strong correlation between two surface derived axes of the distal femur can lead to a false deduction of an optimal reference axis. Since the relationship to the tibia is lacking, conclusions cannot be drawn on the optimal rotational alignment, but only on the morphological description of the distal femur. We believe that the distinction between these two concepts is crucial in the discussion of axial alignment in TKR.

The consequences of our results for surgical practice are as follows. It is obvious that a common frame of reference is to be used if we wish to discuss the achievement of optimal rotation of the femoral component. The definition of the femoral transverse axis as a geometrical reference can support future analysis of deformity in patients. Our study deals only with the first level of error, being the individual variability in angular relationships. The second level of error, the inter- and intrasurgeon variability of finding the landmarks during surgery may be even more important, and should be considered in the overall evaluation of the surgical value in using these landmarks. Siston et $\mathrm{al}^{32}$ studying surgical reliability in determining rotational alignment of the femoral component found that the use of the TRAx (Whiteside's line) produced the largest variability with an SD of $7.6^{\circ}$ and a range of $-12^{\circ}$ to $+15^{\circ}$, with little improvement when the epicondyles were referenced. The lowest range was reported when surgeons used the PCL with a posterior referencing guide $\left(-9^{\circ}\right.$ to $\left.+11^{\circ}\right)$. Middleton and Palmer ${ }^{33}$ reported on the reliability and reproducibility 
of the TRAx in the distal femora in 50 cadavers. A wide range of rotation of the TRAx relative to the surg TEA was reported from $-10^{\circ}$ to $+12^{\circ}$ with an $\mathrm{SD}$ of $4.7^{\circ}$. The ability to mark accurately visually selected anatomical landmarks in a surgical navigation system with a CT scan as a baseline reference has been investigated revealing maximum errors in the TEA in the axial plane of $9.1^{\circ}$ because of registration errors of the medial femoral epicondyle and of $7.2^{\circ}$ due to registration errors of the lateral femoral epicondyle. ${ }^{34}$ This confirms the findings of other reports. ${ }^{35,36}$ Efforts to reduce surgical incisions compromise surgical consistency in locating the TRAx and anat TEA further. ${ }^{37}$

Based on our findings, we believe that the variability of the TRAx is too wide to allow it to serve as a reliable secondary reference during TKR. The additional intra- and intersurgeon variability in the surgical determination of this axis strengthens this. Unfortunately, the latter argument on the difficult intra-operative localisation of landmarks equally applies to the anat TEA and the surg TEA. ${ }^{32,34-38}$ Despite the relatively consistent relationship to the FTAx our findings indicate that surgical precision is insufficient to rely on these axes for positioning the femoral component in the axial plane.

By contrast, the PCL, which had the closest and most constant relationship to the FTAx in our study, is a landmark which can be located accurately and reliably during surgery. ${ }^{9,38}$ It could be used to align the femoral component in the axial plane during surgery, provided that the mean compensation of $1.41^{\circ}$ of external rotation, according to our results, was applied. However, it is important that the surgeon recognises that the articular surface may be eroded due to wear and may induce considerable changes to the described morphology. This would require a further intraoperative compensation if the surgeon relied on the PCL. Recently, Aglietti et a ${ }^{39}$ described a relationship between the orientation of the PCL and coronal alignment, showing more internal rotation in the severe valgus knee and more external rotation in the severe varus knee. The different wear patterns which are observed in the severely deformed varus or valgus knee can certainly explain this. In our series, we found a weak and non-significant correlation $(\mathrm{r}=0.36 ; \mathrm{p}=0.257)$ between the PCL and coronal alignment, allowing us to defend the role of the PCL as a reliable landmark for knees with no or moderate deformity, and without posterior condylar cartilage or bone wear.

No benefits in any form have been received or will be received from a commercial party related directly or indirectly to the subject of this article.

\section{References}

1. Mochizuki RM, Schurman MD. Patellar complications following total knee arthroplasty. J Bone Joint Surg [Am] 1979;61-A:879-83.

2. Rhoads DD, Noble PC, Reuben JD, Mahoney OM, Tullos HS. The effect of femoral component position on patellar tracking after total knee arthroplasty. Clin Orthop 1990;260:43-51.

3. Sikorski JM. Alignment in total knee replacement. J Bone Joint Surg [Br] 2008;90B:1121-7.

4. Berger RA, Crossett LS, Jacobs JJ, Rubash HE. Malrotation causing patellofemoral complications after total knee arthroplasty. Clin Orthop 1998;356:144-53.
5. Miller MC, Berger RA, Petrella AJ, Karmas A, Rubash HE. Optimizing femoral component rotation in total knee arthroplasty. Clin Orthop 2001;392:38-45

6. Freeman MA, Todd RC, Bamert P, Day WH. ICLH arthroplasty of the knee: 19681977. J Bone Joint Surg [Br] 1978;60-B:339-44.

7. Scuderi GR, Insall JN. Posterior stabilized prosthesis. Orthop Clin North Am 1989;20:71-8.

8. Hungerford DS, Kenna RV. Preliminary experience with a total knee prosthesis with porous coating used without cement. Clin Orthop 1983;176:95-107.

9. Laskin RS. Flexion space configuration in total knee arthroplasty. J Arthroplasty 1995; 10:657-60

10. Griffin FM, Insall JN, Scuderi GR. The posterior condylar angle in osteoarthritic knees. J Arthroplasty 1998;13:812-15.

11. Berger RA, Rubash HE, Seel MJ, Warren HT, Crosset LS. Determining the rotational alignment of the femoral component in total knee arthroplasty using the epicondylar axis. Clin Orthop 1993;286:40-7.

12. Asano T, Akagi M, Nakamura T. The functional flexion-extension axis of the knee corresponds to the surgical epicondylar axis. J Arthroplasty 2005;20:1060-7.

13. Poilvache PL, Insall JN, Scuderi GR, Font-Rodriguez DE. Rotational landmarks and sizing of the distal femur in total knee arthroplasty. Clin Orthop 1996;331:35-46.

14. Arima J, Whiteside LA, McCarthy DS, White S. Femoral rotational alignment, based on the anteroposterior axis, in total knee arthroplasty in a valgus knee: a technical note. J Bone Joint Surg [Am] 1995;77-A:1331-4.

15. Moreland JR, Bassett LW, Hanker GJ. Radiographic analysis of the axial alignment of the lower extremity. J Bone Joint Surg [Am] 1987;69-A:745-9.

16. Hanada $\mathbf{H}$, Whiteside LA, Steiger J, Fyer $\mathbf{P}$, Naito $\mathbf{M}$. Bone landmarks are more reliable than tensioned gaps in TKA component alignment. Clin Orthop 2007;462:137-42.

17. Yoshioka Y, Siu D, Cooke TD. The anatomy and functional axes to the femur. $J$ Bone Joint Surg [Am] 1987;69-A:873-80.

18. Griffin MF, Insall JN, Scuderi GR. Accuracy of soft tissue balancing in total knee arthroplasty. J Arthroplasty 2000;15:970-3.

19. Yoshino N, Takai S, Ohtsuki Y, Hirasawa Y. Computed tomography measurement of the surgical and clinical transepicondylar axis of the distal femur in osteoarthritic knees. J Arthroplasty 2001;16:493-7.

20. Iwaki H, Pinskerova V, Freeman MAR. Tibiofemoral movement. 1: the shapes and relative movements of the femur and the tibia in unloaded cadaver knee. J Bone Joint Surg [Br] 2000;82-B:1189-95.

21. Cobb JP, Dixon H, Dandachli W, Iranpour F. The anatomical tibia axis: reliable rotational orientation in knee replacement. J Bone Joint Surg [Br] 2008;90-B:1032-8.

22. LaPrade RF, Engebretsen AH, Ly TV, et al. The anatomy of the medial part of the knee. J Bone Joint Surg [Am] 2007;89-A:2000-10.

23. LaPrade RF, Ly TV, Wentorf FA, Engebretsen L. The posterolateral attachments of the knee: a qualitative and quantitative morphologic analysis of the fibular collateral ligament, popliteus tendon, popliteofibular ligament, and lateral gastrocnemius tendon. Am J Sports Med 2003;31:854-60.

24. Bland JM, Altman DG. Statistical methods for assessing agreement between two methods of clinical measurement. Lancet 1986;1:307-10.

25. Sharkey PF, Hozack WJ, Rothman RH, Shastri S, Jacoby SM. Why are total knee arthroplasties failing today? Clin Orthop 2002;404:7-13

26. Yoshii I, Whiteside LA, White SE, Milliano MT. Influence of prosthetic joint line position on knee kinematics and patellar position. J Arthroplasty 1991;6:169-77.

27. Anouchi YS, Whiteside LA, Kaiser AC, Milliano MT. The effects of axial rotational alignment of the femoral component on knee stability and patellar tracking in total knee arthroplasty demonstrated on autopsy specimens. Clin Orthop 1991;287:170-7

28. Akagi M, Matsuse Y, Mata T, et al. Effect of rotational alignment on patellar tracking in total knee arthroplasty. Clin Orthop 1999;366:155-63.

29. Matsuda S, Miura H, Nagamine R, et al. Effect of femoral and tibial component position on patellar tracking following total knee arthroplasty: 10-year follow-up of Miller-Galante I knees. Am J Knee Surg 2001;14:152-6.

30. Olcott CW, Scott RD. Femoral component rotation during total knee arthroplasty. Clin Orthop 1999;367:39-42

31. Talbot S, Bartlett J. The anterior surface of the femur as a new landmark for femoral component rotation in total knee arthroplasty. Knee Surg Sports Traumatol Arthrosc 2008;16:258-62 
32. Siston RA, Patel JJ, Goodman SB, Delp SL, Giori NJ. The variability of femoral rotational alignment in total knee arthroplasty. J Bone Joint Surg [Am] 2005;87A:2276-80.

33. Middleton FR, Palmer SH. How accurate is Whiteside's line as a reference axis in total knee arthroplasty? Knee 2007;14:204-7.

34. Yau WP, Leung A, Liu KG, Wong LLS, Chiu KY. Interobserver and intra-observer errors in obtaining visually selected anatomical landmarks during registration process in non-image based navigation-assisted total knee arthroplasty. $J$ Arthroplasty 2007;22:1150-61.

35. Jenny JY, Boeri C. Low reproducibility of the intra-operative measurement of the trans-epicondylar axis during total knee replacement. Acta Orthop Scand 2004;75:74-7.
36. Kinzel V, Ledger M, Shakespeare D. Can the epicondylar axis be defined accurately in total knee arthroplasty? Knee 2005;12:293-6.

37. Yau WP, Leung A, Liu KG, et al. Errors in the identification of the transepicondylar and anteroposterior axes of the distal femur in total knee replacement using minimally-invasive and conventional approaches: a cadaver study. J Bone Joint Surg [Br] 2008;90-B:520-6.

38. Galaud B, Beaufils $\mathbf{P}$, Michaut $\mathbf{M}$, et al. Distal femoral torsion: comparison of CT scan and intra-operative navigation instruments during total knee arthroplasty: report of 70 cases. Rev Chir Orthop Reparatrice Appar Mot 2008;94:573-9 (in French).

39. Aglietti P, Sensi L, Cuomo P, Ciardullo A. Rotational position of femoral and tibia components in TKA using the femoral transepicondylar axis. Clin Orthop 2008;466:2751-5 